\title{
EVALUATION OF THE INTERMETATARSAL ANGLE AFTER THE ARTHRODESIS OF THE FIRST METATARSOPHALANGEAL JOINT FOR TREATMENT OF THE HALLUX VALGUS
}

Marco Túlio Costa', Douglas Lobato Lopes Neto², Fábio Henrique Kojima², Ricardo Cardenuto Ferreira ${ }^{3}$

\begin{abstract}
Objective: To evaluate the correction of the intermetatarsal angle after arthrodesis of the metatarsophalangeal joint of the hallux. We believe that varus deformity of the first metatarsal can be corrected after arthrodesis of the first metatarsophalangeal joint, without the need for proximal osteotomy. Methods: Forty-three feet of patients who had undergone arthrodesis of the first metatarsophalangeal joint between May 1997 and October 2009 were retrospectively analyzed by means of radiographs. The mean length of follow-up was 58 months. Measurements on the metatarsophalangeal angle, intermetatarsal angle and sesamoid dislocation were made using radiographs made before, immediately after and later on after the operation. Results: The mean metatarsophalangeal
\end{abstract}

angle was 37.6 degrees preoperatively, 12.8 degrees immediately after the operation and 16.4 degrees later on after the operation. The mean intermetatarsal angle was 16 degrees preoperatively, 10 degrees immediately after the operation and 10.2 degrees later on after the operation. Regarding sesamoid dislocation, preoperative radiographs showed most feet to be classified as G3; immediately after the operation, most were classified as G2; and later on after the operation, most were G1. Conclusion: The intermetatarsal angle and sesamoid dislocation improved with arthrodesis of the first metatarsophalangeal joint, without the need for osteotomy at the base of the first metatarsal.

Keywords - Arthrodesis; Metatarsophalangeal Joint; Hallux Valgus

\section{INTRODUCTION}

Hallux valgus is characterized by lateral deviation of the hallux, as measured on radiographic examinations by the metatarsophalangeal angle, and by medial deviation of the head of the metatarsal, as measured on radiographs by the intermetatarsal angle ${ }^{(1-3)}$. Surgical treatment is recommended for correcting the painful deformities. In cases in which the deformity is considered to be moderate or severe, a proximal approach to the first metatarsal is recommended, with the aim of correcting the varus deformity of this bone. Arthrodesis of the first metatarsophalangeal joint is a treatment option for patients with severe hallux valgus, recurrent hallux valgus and deformities secondary to inflammatory arthritis of the first metatarsophalangeal joint, such as in cases of rheumatoid arthritis ${ }^{(4-10)}$.

Since arthrodesis does not directly address varus deformity of the first metatarsal, there are doubts in relation to patients with an increased intermetatarsal angle regarding whether the varus deformity can be corrected by means of arthrodesis of the first metatarsophalangeal joint alone, or whether osteotomy at the base of the first metatarsal is necessary ${ }^{(11,12)}$. Many techniques for arthrodesis of the first metatarsophalangeal joint have been described in the literature, but few authors have investigated the change in intermetatarsal angle after arthrodesis ${ }^{(11,13-16)}$.

Our hypothesis was that varus deformity of the first metatarsal becomes corrected through arthrodesis of

1 - Attending Physician in the Foot and Ankle Group, Department of Orthopedics and Traumatology, Santa Casa de Misericórdia de São Paulo, São Paulo, SP, Brazil.

2 - Resident Physician in the Department of Orthopedics and Traumatology, Santa Casa de Misericórdia de São Paulo, São Paulo, SP, Brazil.

3 - Head of the Foot and Ankle Group, Santa Casa de Misericórdia de São Paulo, São Paulo, SP, Brazil.

Work performed in the Department of Orthopedics and Traumatology, School of Medical Sciences, Santa Casa de São Paulo.

Correspondence: Departamento de Ortopedia de Traumatologia, Rua Cesário Mota Junior 112, 01224-000 São Paulo, SP. E-mail: tuliom@uol.com.br

Work received for publication: July 21, 2011; accepted for publication: September 12, 2011.

The authors declare that there was no conflict of interest in conducting this work 
the first metatarsophalangeal joint, without the need for proximal osteotomy. To prove this hypothesis, we studied a series of radiographs on patients who underwent arthrodesis on the first metatarsophalangeal joint of the hallux, comparing the angles of the hallux valgus, intermetatarsal angle and dislocation of the sesamoids, on preoperative, immediate postoperative and later postoperative radiographs. The objective of this study was to evaluate the radiographic correction of the intermetatarsal I-II angle following arthrodesis of the metatarsophalangeal joint of the hallux.

\section{MATERIAL AND METHOD}

We retrospectively analyzed 43 radiographs on the feet of 35 patients who underwent arthrodesis of the metatarsophalangeal joint of the hallux, between May 1997 and October 2009, performed by the Foot and Ankle Group of Santa Casa de Misericórdia de São Paulo. Patients with a previous history of surgery on the forefoot or who evolved with pseudarthrosis were excluded from this study. The patients' mean aged at the time of the surgery was 65 years (ranging from 22 to 86 years). There were seven male patients $(20 \%)$ and 28 females $(80 \%)$. Twenty-seven patients $(74.3 \%)$ had rheumatoid arthritis and nine $(25.7 \%)$ presented severe degeneration of the metatarsophalangeal joint of the hallux. The mean length of follow-up was 58 months (ranging from 12 to 125 months). Arthrodesis was performed by using a dorsal access route. The fixation was done using a dorsal plate and screws in 31 feet $(72 \%)$ of 26 patients (20 of these patients had a diagnosis of rheumatoid arthritis) or two Kirschner wires, in 12 feet $(28 \%)$ of nine patients (six of them with rheumatoid arthritis).

To evaluate the angular correction of the deformities, we measured the angle between the first and second metatarsals (intermetatarsal angle), the angle between the first metatarsal and the proximal phalange of the hallux (metatarsophalangeal or hallux valgus angle) and the position of the sesamoids (dislocation of the sesamoids) in relation to the head of the first metatarsal. We used radiography in dorsoplantar view, with weight-bearing, produced before the operation, during the immediate postoperative period (without weight-bearing, in the surgical theater) and at a later time after the operation (at the final outpatient consultation). The intermetatar- sal angle, metatarsophalangeal angle (hallux valgus angle) and dislocation of the sesamoids were measured, by two of the authors, independently. The values used in this study were the averages from the two measurements thus obtained. We used the criteria recommended by the American Orthopaedic Foot \& Ankle Society for measuring the angles ${ }^{(17)}$. The reference points for the first and second metatarsals were placed one to two centimeters from the proximal and distal joint surfaces, at the center of the diaphysis. For the proximal phalange, they were placed 0.5 to 1.0 centimeters from the proximal and distal joint surfaces (Figure 1). Dislocation of the sesamoids was assessed in terms of the position of the tibial sesamoid in relation to the mechanical axis of the first metatarsal (Figure 2), and was classified into four types: grade 0 - the tibial sesamoid was located medially to the mechanical axis that occupied the space between the two ossicles; grade 1 - the tibial sesamoid was cut by the axis used, but in a proportion less than $50 \%$ of its width; grade 2 - the tibial sesamoid was cut by the axis used and more than $50 \%$ of its total mass was lateralized; and grade 3 - when complete lateral dislocation of the glenosesamoid apparatus occurred ${ }^{(18)}$.

For the statistical analysis, all the variables were firstly analyzed descriptively. For the quantitative variables, this analysis was done by means of observing minimum and maximum values and calculating means, standard deviations and medians. For the qualitative variables, absolute and relative frequencies were calculated. To compare the preoperative, immediate postoperative and late postoperative times, the Friedman nonparametric test was used, since the assumption of normal distribution of the data was rejected. The software used was SPSS 15.0 for Windows. The significance level used for the tests was $5 \%{ }^{(19)}$.

\section{RESULTS}

Table 1 shows that there was a mean reduction in the hallux valgus angle of 24.8 degree, comparing the preoperative and immediate postoperative angles $(\mathrm{p}<0.05)$, and a reduction of 21.2 degrees, comparing the preoperative and late postoperative angles $(p<0.05)$. There was an increase in the angle of 3.6 degrees, comparing the immediate and late postoperative angles $(\mathrm{p}>0.05)$.

Table 1 also shows that there was a mean reduction 


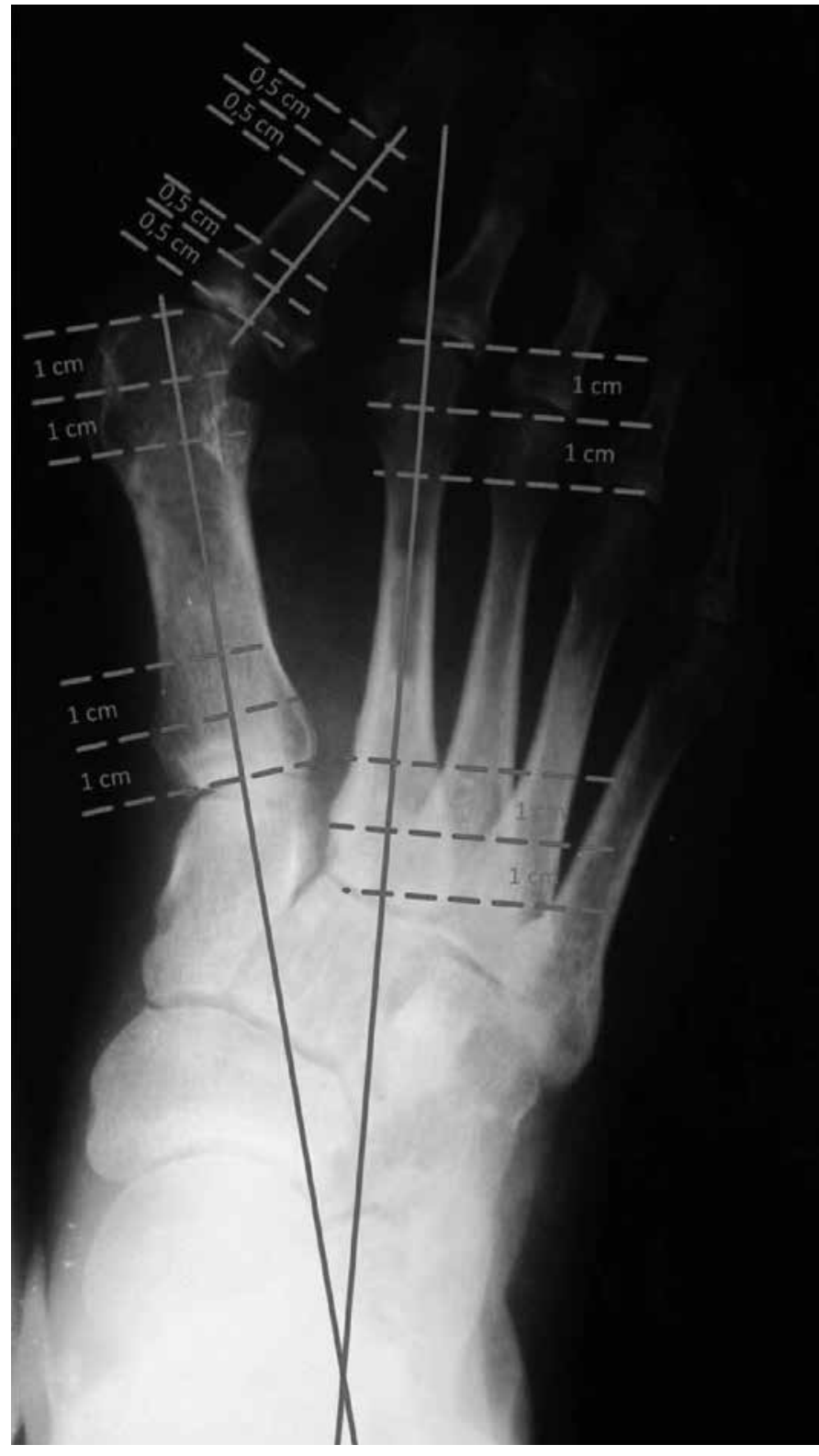

Figure 1 - Location of the reference points for the first and second metatarsals: 1 to $2 \mathrm{~cm}$ from the proximal and distal joint surfaces of each bone. Because of the shorter length of the phalange, the reference points were placed 0.5 to $1 \mathrm{~cm}$ from the proximal and distal surfaces of the proximal phalange of the hallux.

in the intermetatarsal angle of 6.0 degrees, comparing the preoperative and immediate postoperative angles $(\mathrm{p}<0.05)$ and a reduction of 5.8 degrees, comparing the preoperative and late postoperative angles $(\mathrm{p}<0.05)$. There was an increase in the angle of 0.2 degrees, comparing the immediate and late postoperative angles $(\mathrm{p}>0.05)$.

Table 2 shows that the greatest number of feet was classified as G3 on preoperative radiographs, while the greatest number of feet was classified as G2 on immediate postoperative radiographs and as G1 on late postoperative radiographs.

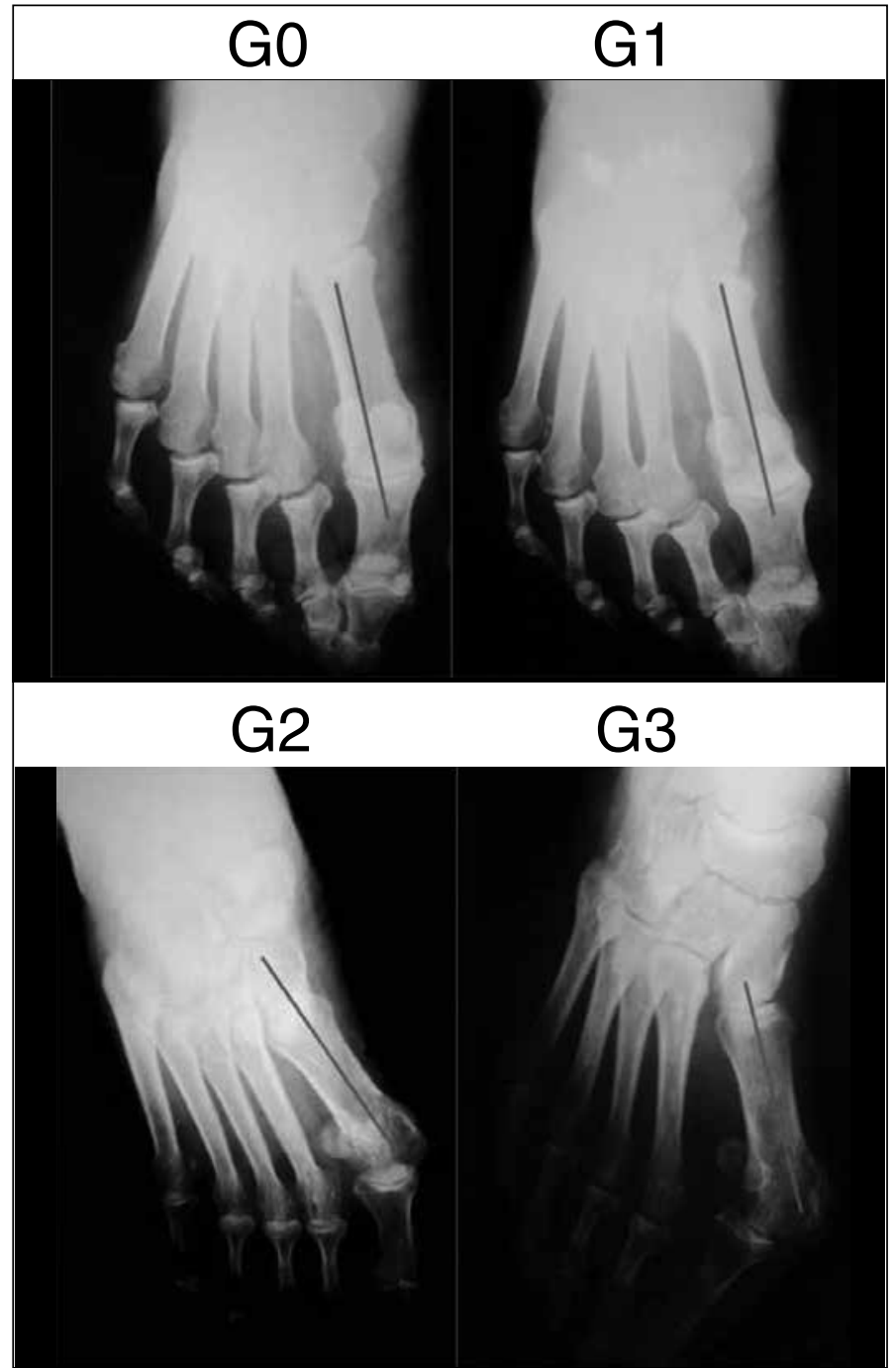

Figure 2 - Severity of dislocation of the sesamoids.

$\mathrm{GO}$ - the tibial sesamoid was located medially to the mechanical axis that occupied the space between the two ossicles; G1 - the tibial sesamoid was cut by the axis used, but in a proportion less than $50 \%$ of its width; G2 - the tibial sesamoid was cut by the axis used and more than $50 \%$ of its total mass was lateralized; and G3 - when complete lateral dislocation of the glenosesamoid apparatus occurred.

Table 1 - Mean values for the hallux valgus and intermetatarsal angles on preoperative, immediate postoperative and late postoperative radiographs.

\begin{tabular}{c|c|c|c}
\hline & PRE & IPO & LPO \\
\hline HV angle & 37.6 & 12.8 & 16.4 \\
\hline IM angle & 16.0 & 10.0 & 10.2 \\
\hline \multicolumn{2}{l|}{ HV - hallux valgus; IM - intermetatarsal; PRE - preoperative; IPO - immediate postoperative; }
\end{tabular}
LPO - late postoperative.

Table 2 - Mean values for dislocation of the sesamoids on preoperative, immediate postoperative and late postoperative radiographs.

\begin{tabular}{c|c|c|c}
\hline & PRE & IPO & LPO \\
\hline G0 & 3 & 6 & 7 \\
\hline G1 & 7 & 11 & 21 \\
\hline G2 & 3 & 23 & 13 \\
\hline G3 & 30 & 3 & 2 \\
\hline Total & 43 & 43 & 43
\end{tabular}

G0 - grade 0; G1 - grade 1; G2 - grade 2; G3 - grade 3; PRE - number of feet before operation; IPO - number of feet immediately after the operations; POT - number of feet later on after the operation. 


\section{DISCUSSION}

Arthrodesis of the first metatarsophalangeal joint is considered to be the gold-standard technique for treating patients with severe hallux valgus, recurrent hallux valgus and deformities secondary to inflammatory arthritis of the hallux ${ }^{(4-10,20,21)}$. In a prospective study, Brodsky et $a l^{(6)}$ showed that arthrodesis of this joint improved the stability and propulsive force during gait, for feet with severe joint degeneration. Many techniques for performing arthrodesis of the first metatarsophalangeal joint have been described, with consolidation rates ranging from 80 to $100 \%{ }^{(5-10)}$. Biomechanical studies using different types of fixation have shown that the most stable fixation method is one that combines a traction screw and a dorsal plate ${ }^{(22,23)}$. In evaluating the intermetatarsal angle, we found that the mean was 10 degrees on immediate postoperative radiographs and 10.2 degrees on late postoperative radiographs. These data show that the varus deformity of the first metatarsal can be corrected by means of arthrodesis on the first metatarsophalangeal joint in patients with hallux valgus, without the need for osteotomy at the base of the first metatarsal. We found that there was a mean correction of 6.0 degrees, comparing the preoperative and immediate postoperative radiographs, and 5.8 degrees, comparing the preoperative and late postoperative radiographs. Our data corroborate the findings of other authors, in which the mean corrections were $5.7^{(13)}, 4^{(14)}, 4.4^{(15)}, 4.4^{(12)}$ and $8.2^{(11)}$. It is believed that the alignment of the first radius restores the anatomical relationships and function of the intrinsic and extrinsic musculature and plantar aponeurosis. This leads to diminished mobility of the metatarsocuneiform joint, thus achieving correction of the intermetatarsal angle following arthrodesis of the first metatarsophalangeal joint ${ }^{(24)}$. In our study, we observed a small difference of 0.2 degrees $(p>0.05)$ in the mean intermetatarsal angle, comparing the immediate postoperative radiograph (obtained without weight-bearing) and the late postoperative radiograph (obtained with weight-bearing. Considering that there was no statistically significant difference between the measurements of this angle on the two radiographs, we believe that the angle measured on an immediate postoperative radiograph without weight-bearing will be similar to the angle measured on a late postoperative radiograph, pro- duced with weight-bearing. Based on these results, we can affirm that the final radiographic correction of this angle can be safely determined during the operation, with a radiograph produced without weight-bearing. We believe that the difference of 0.2 degrees that we found could have occurred due to manual measurement of these angles.

The mean hallux valgus angle in the immediate postoperative period was 12.8 degrees, which is considered normal ${ }^{(25)}$, representing a mean reduction of 24.8 degrees $(p<0.05)$, comparing the preoperative and immediate postoperative radiographs. We also observed that there was a loss of 3.6 degrees $(\mathrm{p}>0.05)$, comparing the immediate and late postoperative radiographs. Although the immediate postoperative radiograph was produced without weight-bearing while the late postoperative radiograph was produced with weight-bearing, we do not believe that this could have been the cause of the angular difference observed between them. In our view, this loss (without statistical significance: $p>0.05$ ) occurred because of the fixation with Kirschner wires that was used on some feet, a method that provides less stability ${ }^{(22,23)}$. Kirschner wires were chosen based on the bone quality observed by the surgeon during the operation. In some of the cases that we studied, the patients presented rheumatoid arthritis and were using corticoids, which is known to lead to osteoporosis, thus making fixation with a plate and screws difficult ${ }^{(4,20,21)}$. Because of these results, we recommend that fixation of arthrodesis should be done using a plate and screws, whenever possible, so as to obtain better stability.

Few authors have yet compared dislocation of the sesamoids after arthrodesis of the metatarsophalangeal joint of the hallux ${ }^{(11)}$. In our sample, we observed improvement of the dislocation of the sesamoids, and this was proven by the increase in the number of feet classified as mild cases (G0 and G1) and a decrease in the number of feet classified as moderate to severe cases (G2 and G3), comparing the preoperative, immediate postoperative and late postoperative radiographs on these patients. We believe that restoration of soft-tissue balance, in association with alignment of the first radius following arthrodesis of the first metatarsophalangeal joint, is responsible for the observed improvement in dislocation of the sesamoids in our study. 


\section{CONCLUSION}

Based on this study, we observed that the intermetatarsal angle and the dislocation of the sesamoids improved through arthrodesis of the first metatarsophalangeal joint, without the need for osteotomy at the base of the first metatarsal to correct the intermetatarsal angle.

\section{REFERENCES}

1. Nery C. Hálux valgo. Rev Bras Ortop. 2001;36(6):183-200.

2. Prado Junior I, Gomes SMP, Rezende RR. Estudo radiográfico, na incidência ântero-posterior, dos ângulos intermetatarsiano I-II, articular metatarsiano distal I e articular proximal da falange proximal do hálux, em pés normais de adultos. Rev Bras Ortop. 1999;34(8):489-94.

3. Salomão O. Hálux valgo: etiologia e tratamento. Rev Bras Ortop. 2005; 40(4):147-52.

4. Mann RA, Thompson FM. Arthrodesis of the first metatarsophalangeal joint for hallux valgus in rheumatoid arthritis. 1984. Foot Ankle Int. 1997;18(2):65-7.

5. Bennett GL, Kay DB, Sabatta J. First metatarsophalangeal joint arthrodesis: an evaluation of hardware failure. Foot Ankle Int. 2005;26(8):593-6.

6. Brodsky JW, Baum BS, Pollo FE, Mehta H. Prospective gait analysis in patients with first metatarsophalangeal joint arthrodesis for hallux rigidus. Foot Ankle Int. 2007;28(2):162-5.

7. Brodsky JW, Passmore RN, Pollo FE, Shabat S. Functional outcome of arthrodesis of the first metatarsophalangeal joint using parallel screw fixation. Foot Ankle Int. 2005;26(2):140-6.

8. Flavin R, Stephens MM. Arthrodesis of the first metatarsophalangeal joint using a dorsal titanium contoured plate. Foot Ankle Int. 2004;25(11):783-7.

9. Goucher NR, Coughlin MJ. Hallux metatarsophalangeal joint arthrodesis using dome-shaped reamers and dorsal plate fixation: a prospective study. Foot Ankle Int. 2006;27(11):869-76.

10. Kumar S, Pradhan R, Rosenfeld PF. First metatarsophalangeal arthrodesis using a dorsal plate and a compression screw. Foot Ankle Int. 2010;31(9):797-801.

11. Cronin JJ, Limbers JP, Kutty S, Stephens MM. Intermetatarsal angle after first metatarsophalangeal joint arthrodesis for hallux valgus. Foot Ankle Int. 2006;27(2):104-9.

12. Pydah SK, Toh EM, Sirikonda SP, Walker CR. Intermetatarsal angular change following fusion of the first metatarsophalangeal joint. Foot Ankle Int. 2009;30(5):415-8.
The radiographs produced during the immediate postoperative period without weight-bearing in our study were useful for assessing the final intermetatarsal angle, and these were as trustworthy as the radiographs produced with weight-bearing in the late postoperative period.
13. Humbert $\mathrm{JL}$, Bourbonnière $\mathrm{C}$, Laurin $\mathrm{CA}$. Metatarsophalangeal fusion for hallux valgus: indications and effect on the first metatarsal ray. Can Med Assoc J. 1979;120(8):937-41, 956.

14. Riggs SA Jr, Johnson EW Jr. McKeever arthrodesis for the painful hallux. Foot Ankle. 1983;3(5):248-53.

15. Mann RA, Katcherian DA. Relationship of metatarsophalangeal joint fusion on the intermetatarsal angle. Foot Ankle. 1989;10(1):8-11.

16. von Salis-Soglio G, Thomas W. Arthrodesis of the metatarsophalangeal joint of the great toe. Arch Orthop Trauma Surg. 1979;95(1-2):7-12.

17. Coughlin MJ, Saltzman CL, Nunley JA 2nd. Angular measurements in the evaluation of hallux valgus deformities: a report of the ad hoc committee of the American Orthopaedic Foot \& Ankle Society on angular measurements. Foot Ankle Int. 2002;23(1):68-74.

18. Smith RW, Reynolds JC, Stewart MJ. Hallux valgus assessment: report of research committee of American Orthopaedic Foot and Ankle Society. Foot Ankle.1984;5(2):92-103.

19. Rosner B. Fundamentals of biostatistics. 2nd ed. Boston: PWS Publishers; 1986.

20. Coughlin MJ. Rheumatoid forefoot reconstruction. A long-term follow-up study. J Bone Joint Surg Am. 2000;82(3):322-41.

21. Fuhrmann RA. [The treatment of rheumatoid foot deformities]. Orthopade. 2002;31(12):1187-97.

22. Molloy S, Burkhart BG, Jasper LE, Solan MC, Campbell JT, Belkoff SM. Biomechanical comparison of two fixation methods for first metatarsophalangeal joint arthrodesis. Foot Ankle Int. 2003;24(2):169-71.

23. Politi J, John H, Njus G, Bennett GL and Kay DB. First metatarsal-phalangeal joint arthrodesis: a biomechanical assessment of stability. Foot Ankle Int. 2003;24(4):332-7.

24. Coughlin MJ, Jones CP. Hallux valgus and first ray mobility. A prospective study. J Bone Joint Surg Am. 2007;89(9):1887-98.

25. Hardy $\mathrm{RH}$, Clapham JC. Observations on hallux valgus; based on a controlled series. J Bone Joint Surg Br. 1951;33(3):376-91. 